that many of these short comings are associated with the decreasing number of consultant oral microbiologists (COMs). COMs have the necessary training and experience to teach undergraduates and postgraduates the prevention, diagnosis, surveillance and management of oral and maxillofacial infections in addition to the important principles of antimicrobial stewardship. The latter is essential to ensure the appropriate use of antimicrobial agents both in limiting serious side effects and reducing the emergence and spread of antibiotic resistance. The key requirements of 'high calibre skills base' and 'modernising the surveillance of drug resistance' have been recently highlighted in the 0'Neill report ${ }^{2}$ on the Global Health Crisis caused by antimicrobial resistance.

The dental profession has relied too long on the assumptions that the microbiology of oral and maxillofacial infections is readily predictable, pathogens are always susceptible to first line antimicrobial agents and that oral and maxillofacial infections are associated with negligible adverse patient outcomes. Today, there is evidence to suggest that even metronidazole may be losing its effectiveness for some species of anaerobes. ${ }^{3}$

At present, ACOM is engaged with the Department of Health in the drafting of a guide for commissioners for the supporting specialties. This is a critical opportunity to recruit and train the next generation of dentally qualified COMs. Two of the biggest challenges to the dental profession over the last decade have included instrument decontamination and antimicrobial stewardship. There are currently too few COMs to adequately address these issues. ACOM proposes that a network of increased numbers of COMs is established as soon as possible to provide leadership in the management of these and other issues.

The need for COMs will increase further with the greater use of implantable biotechnologies and an ageing population with multiple co-morbidities predisposed to infection with more unusual and pan-resistant microorganisms. Advances in diagnostic technologies that increase our understanding of the oral microbiome will be accompanied by a need for clinical skills that integrate clinical microbiology and in-depth knowledge of the complexity of the oral microflora and its increasingly apparent role in systemic diseases. COMs are ideally placed to provide their expertise and leadership through further training at a specialist level but this requires the creation of the appropriate number of training and consultant posts.

C. Pankhurst, R. Rautemaa-Richardson,

N. Seoudi, A. Smith, M. Wilson, by email DOI: $10.1038 /$ sj.bdj.2016.5
1. Beacher, N, Sweeney M P, Bagg J. Dentists, antibiotics and Clostidium difficle-associated disease. Br Dent J 2015; 219: 275-279.

2. Tackling a Global Health Crisis: initial steps. The review on Antimicrobial Resistance chaired by Jim O'Neill. February 2015. Available online at: www. amr-review.org/sites/default/files/RARJ3003_ Global_health_crisis_report_20.03.15_OUTLINED. pdf (Accessed January 2016).

3. Veloo A C M, Boiten K E, Wekema-Mulder G J et al., Antibiotic susceptibility profiles of Prevotella species in the Netherlands. Int J Antimicrob Agents 2015; 45: 554-556.

\section{RIGHT OF REPLY}

\section{A proud contribution}

Sir, we read with concern the recent Opinion article regarding our arginine dentifrice research programme (Shaw et al. BDJ 2015; 219: 567-569). As a company dedicated to improving oral health globally, we strongly disagree with the opinions expressed and the conclusions and implications drawn in the article. We are proud of the contribution we have made to caries prevention and the research programme supporting it. The company's detailed response to the opinion article can be viewed at www.colgateprofessionaluk.co.uk/Full-commentary-re-BDJ18-12-2015-opinion-article.html.

We especially want to call out three important facts:

1. The suggestion by the reviewers that claims of efficacy are unfounded and misleading is completely unsupported and based on a limited number of the published studies. The studies sponsored by Colgate and conducted by respected clinical researchers demonstrate that fluoride toothpaste with 1.5\% arginine represents a new standard of care and a step change in caries prevention from conventional fluoride toothpaste. The concerns raised by the reviewers regarding a lack of detail presented in study reports are not evidence of errors in study design. The research programme consists of nine clinical efficacy studies, published in four peer-reviewed journals, conducted in collaboration with leading dental universities around the world and involved more than 16,000 participants. They demonstrate that the arginine formula, compared to regular fluoride toothpaste, reduces new cavities by up to $20 \%$ at two years. ${ }^{1,2}$ In addition, the clinical programme has shown that brushing with arginine toothpastes contributes to four times greater remineralisation than a conventional fluoride toothpaste and reverses early caries lesions by approximately half in six months. Furthermore, a community-based study conducted in Thailand demonstrated up to 40\% reductions in dental decay when arginine toothpaste was incorporated into a school based oral health programme $^{3}$

2. For the two studies that the reviewers questioned the use of non-fluoride toothpaste, the protocols and their ethics were reviewed by the Institutional Review Board (Ethics Committee) of Sichuan University, home to one of China's leading dental schools. It is this review board that is best qualified to judge the ethics of a research rogramme in China. The review recognised the compelling reason to include a nonfluoride control group in the study, to gather direct evidence of fluoride toothpaste's efficacy in the Chinese setting and thereby increase acceptance of fluoride as the standard of care in China. Given this compelling rationale and the robust measures taken to ensure all study participants enjoyed equal long-term oral health benefits, the Institutional Review Board judged that a non-fluoride control was justified under Article 33 of the Helsinki Declaration

3. Contrary to what is suggested in the Opinion article, all authors with an affiliation to the Colgate-Palmolive Company were acknowledged and sponsorship by the company was made clear. We recognise that the potential for bias exists in industrysponsored research, as it does for academic research. In all cases, we should rely on established processes, including publication in peer reviewed journals, to ensure the validity and reliability of research results.

Colgate-Palmolive believes in robust criticism that is based on facts and conducted with the goal of improving research outcomes. With such review, industry will maintain its ability to prove and publish on innovative technologies that benefit the public's oral health particularly those that impact children.

W. DeVizio and R. Ellwood
Colgate-Palmolive Company,
New Jersey, USA
DOI: $10.1038 /$ sj.bdj.2016.6

1. Kraivaphan et al. Two-year caries clinical study of the efficacy of novel dentifrices containing 1.5\% arginine, an insoluble calcium compound and 1,450 ppm fluoride. Caries Res 2013; 47: 582-590.

2. Li et al. Arginine promotes fluoride uptake into artificial carious lesions in vitro. Aust Dent J 2015; 60: 104-111.

3. Petersen et al., School-based intervention for improving the oral health of children in southern Thailand. 2015; 32: 44-50. 